\title{
Guest Editorial for Special Issue Application of Concurrency to System Design
}

This special issue entitled "Application of Concurrency to System Design" is devoted to extended versions of selected contributions from the technical sessions of the 14th International Conference on Application of Concurrency to System Design (ACSD) that was held in Tunis, Tunisia, from June 25 to 27, 2014. The aim of the ACSD conference is to bring together researchers and practitioners in the areas of design, modeling, and algorithms for concurrent hardware and software systems, with particular focus on case studies related to the design of embedded systems.

The ACSD 2014 program committee included 49 researchers from 19 countries. Each of the 50 submitted papers was evaluated by at least three reviewers. Afterward, reports were returned to the committee for discussion and resolution of conflicts. Based on their recommendations, 18 regular and 2 tool papers were selected for presentation at the conference. The proceedings including these accepted papers were published by IEEE Xplore. After ACSD 2014, authors of the 10 best papers were invited to submit extended versions of their work to this special issue. The call for papers was open, and 20 submissions were eventually received. After additional refereeing and further revisions, the guest editors were able to accept the following 7 papers for the inclusion in this special issue.

The first article, "Memory-Model-Aware Testing: A Unified Complexity Analysis" by Florian Furbach, Roland Meyer, Klaus Schneider, and Maximilian Senftleben, tackles the problem of analysis of programs running on multiprocessor architectures with weak memory consistency models. The authors focus on the testing approach to program analysis and determine the complexity of the testing problem for a large number of known memory models. To avoid the explicit consideration of each model, the authors employ the Steinke-Nutt hierarchy of memory models. This allows covering ranges (with respect to the hierarchy) of models with a single reduction, thereby significantly simplifying the presented proofs.

The second article, "Action Synthesis for Branching Time Logic: Theory and Applications" by Michał Knapik, Artur Męski, and Wojciech Penczek, presents pmARCTL-a parametric extension of Action-Restricted Computation Tree Logic. The authors give a formal definition for pmARCTL and its semantics, discuss the associated complexity results (in particular, proving that the emptiness problem for pmARCTL is NP-complete), and present a practical parameter synthesis algorithm for pmARCTL that significantly outperforms a naive solution.

The third article, "Parametrised Modal Interface Automata" by Antti Siirtola and Keijo Heljanko, presents the first interface theory that allows specifying a parametric number of interfaces, which is essential for the analysis of real-life systems comprising components, the number of which cannot be fixed a priori. The authors also provide an algorithmic procedure implemented in a software tool for checking the compatibility and refinement between parameterised interfaces. It is demonstrated that the presented algorithm is in some sense close to the decidability frontier-that is, if one lifts some of the used restrictions, the problem becomes undecidable.

The fourth article, "STM-HRT: A Robust and Wait-Free STM for Hard RealTime Multicore Embedded Systems” by Audrey Queudet, Sylvain Cotard, Jean-Luc

(c) 2015 ACM 1539-9087/2015/10-ART62 $\$ 15.00$

DOI: http://dx.doi.org/10.1145/2809925 
Béchennec, Sébastien Faucou, and Yvon Trinquet, introduces a nonblocking wait-free software transactional memory (STM) protocol for hard real-time (HRT) multicore embedded systems. STM-HRT is a robust protocol in the sense that it avoids fault propagation when a processor or a task fails. The authors' emphasis on the high dependability of the developed protocol is motivated by the automotive application. To ensure the correctness of the protocol, formal verification techniques are employed.

The fifth article, "Failure Semantics for Modal Transition Systems" by Ferenc Bujtor and Walter Vogler, defines a new refinement preorder for modal transition systems based on a variant of the testing approach introduced by De Nicola and Hennessy. The authors demonstrate that in contrast to standard modal refinements, the new refinement preorder "supports itself." Furthermore, the new refinement gives rise to the conjunction operator that is closed on modal transition systems-an important property that is violated by the standard notions of refinement. The authors conclude the article with an illustration of the relations between different refinement preorders.

The sixth article, "Incremental Analysis of Cyclo-Static Synchronous Dataflow Graphs" by Robert de Groote, Philip Hölzenspies, Jan Kuper, and Gerard Smit, presents a semantics for synchronous dataflow graphs (SDFs), which leads to transformation techniques between variants of SDF, and to algorithms for their analysis, combining known methods. In particular, the approach proposed by the authors allows the designer to choose between exact analysis techniques and approximate techniques, finding the best trade-off between cost and accuracy.

The seventh article, "Diagnosability under Weak Fairness" by Vasileios Germanos, Stefan Haar, Victor Khomenko, and Stefan Schwoon, deals with the diagnosis of discrete event systems. After illustrating through an example that the original definition of weak fair diagnosability in partially observed Petri nets had a major flaw, the authors present a correct and efficient framework allowing the verification of weak fair diagnosability in partially observed Petri nets.

Kamel Barkaoui Cedric Conservatoire National des Arts et Métiers, France

Luca Bernardinello Università degli studi di Milano-Bicocca, Italy

Andrey Mokhov Newcastle University, United Kingdom

Guest Editors 